\title{
Metabolic syndrome as a risk factor for total hip or knee replacement due to primary osteoarthritis: a prospective cohort study (the HUNT study and the Norwegian Arthroplasty Register)
}

This article was published in the following Dove Press journal:

Clinical Epidemiology

\begin{abstract}
Alf Inge Hellevik, 1,2 Marianne
Bakke Johnsen, ${ }^{3,4}$ Arnulf Langhammer, ' Valborg Baste, ${ }^{5}$ Ove Furnes, ${ }^{6,7}$ Kjersti Storheim, ${ }^{3,4}$ John Anker Zwart, ${ }^{3,4}$ Gunnar Birkeland Flugsrud, ${ }^{2}$ Lars Nordsletten, ${ }^{2,4}$

'The HUNT Research Centre, Department of Public Health and Nursing, Faculty of Medicine and Health Sciences, NTNU, Norwegian University of Science and Technology, Levanger, ${ }^{2}$ Division of Orthopaedic Surgery, Oslo University Hospital, Oslo, ${ }^{3}$ Research and Communication Unit for Musculoskeletal Health, Division of Clinical Neuroscience, Oslo University Hospital, Oslo, ${ }^{4}$ Faculty of Medicine, University of Oslo, Oslo, ${ }^{5}$ Uni Research Health, Bergen, ${ }^{6}$ The Norwegian Arthroplasty Register, Department of Orthopedic Surgery, Haukeland University Hospital, Bergen, ${ }^{7}$ Department of Clinical Medicine, Institute of Medicine and Dentistry, University of Bergen, Bergen, Norway
\end{abstract}

Video abstract

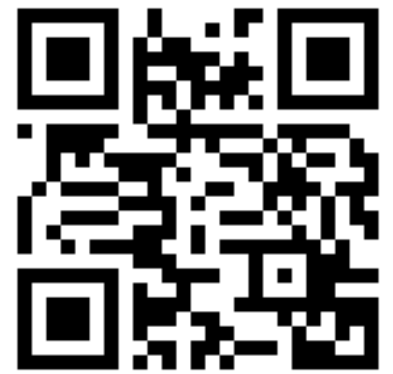

Point your SmartPhone at the code above. If you have a QR code reader the video abstract will appear. Or use: http://youtu.be/labBIV_4t-0

Correspondence: Alf Inge Hellevik

The HUNT Research Centre, Forskningsveien 2

$\mathrm{N}-7600$ Levanger, Norway

$\mathrm{Tel}+4741335383$

Email alf.hellevik@ntnu.no
Objective: Biochemical changes associated with obesity may accelerate osteoarthritis beyond the effect of mechanical factors. This study investigated whether metabolic syndrome and its components (visceral obesity, hypertension, dyslipidemia and insulin resistance) were risk factors for subsequent total hip replacement (THR) or total knee replacement (TKR) due to primary osteoarthritis.

Design: In this prospective cohort study, data from the second survey of the Nord-Trøndelag Health Study 2 (HUNT2) were linked to the Norwegian Arthroplasty Register for identification of the outcome of THR or TKR. The analyses were stratified by age $(<50,50-69.9$ and $\geq 70$ years) and adjusted for gender, body mass index, smoking, physical activity and education.

Results: Of the 62,661 participants, 12,593 (20.1\%) were identified as having metabolic syndrome, and we recorded 1,840 (2.9\%) THRs and 1,111 (1.8\%) TKRs during a mean follow-up time of 15.4 years. Cox regression analyses did not show any association between full metabolic syndrome and THR or TKR, except in persons $<50$ years with metabolic syndrome who had a decreased risk of THR (hazard ratio [HR] 0.58, 95\% CI 0.40-0.83). However, when including only participants whose exposure status did not change during follow-up, this protective association was no longer significant. Increased waist circumference was associated with increased risk of TKR in participants <50 years (HR 1.62, 95\% CI 1.10-2.39) and 50-69.9 years (HR $1.43,95 \%$ CI $1.14-1.80$ ). Hypertension significantly increased the risk of TKR in participants $<50$ years (HR 1.38, 95\% CI 1.05-1.81), and this risk was greater for men.

Conclusion: This study found an increased risk of TKR in men $<50$ years with hypertension and persons $<70$ years with increased waist circumference. Apart from this, neither metabolic syndrome nor its components were associated with increased risk of THR or TKR due to primary osteoarthritis.

Keywords: osteoarthritis, metabolic syndrome, total hip replacement, total knee replacement

\section{Introduction}

Hip and knee osteoarthritis cause significant morbidity and disability in a large proportion of the population. ${ }^{1}$ There is no curative treatment for osteoarthritis, and this places the emphasis on identifying preventable risk factors. Increased body mass index (BMI) is a well-established risk factor for osteoarthritis, both in the knee ${ }^{2-4}$ and the hip. ${ }^{5-7}$ However, biochemical changes associated with obesity may accelerate osteoarthritis beyond the effect of mechanical factors. ${ }^{8,9}$ Metabolic osteoarthritis has, therefore, been suggested as a subtype of osteoarthritis, and links between this phenotype and metabolic syndrome have been reported. ${ }^{10,11}$ 
Metabolic syndrome is a cluster of components associated with increased risk of cardiovascular disease. ${ }^{12}$ These include increased waist circumference, high blood pressure, elevated triglycerides, reduced high-density lipoprotein (HDL) and elevated serum glucose or diabetes. Due to the high prevalence of these components among persons with osteoarthritis, it has been suggested that metabolic syndrome may influence the development of osteoarthritis independent of BMI. ${ }^{13,14}$ This could be explained by shared mechanisms in the etiologies of osteoarthritis and metabolic syndrome: inflammation, oxidative stress, common metabolites and endothelial dysfunction. ${ }^{11}$ However, it is possible that osteoarthritis and metabolic syndrome simply coexist through their common shared risk factors of age and obesity. ${ }^{15}$

The results of observational studies in humans have been inconsistent. One Australian prospective cohort study found that a cumulative number of metabolic syndrome components, central obesity and hypertension were associated with increased risk of total knee replacement (TKR) due to osteoarthritis independent of BMI, but no associations were observed for total hip replacement (THR). ${ }^{15}$ However, the Malmö Diet and Cancer Study found that only central obesity was associated with increased risk of knee osteoarthritis independent of BMI. ${ }^{16}$ Metabolic syndrome and its components were not associated with hip osteoarthritis. Other studies have also reported an increased risk of knee osteoarthritis associated with an increase in the number of metabolic syndrome components. ${ }^{17}$ In contrast, a recent study reported that, after adjustment for BMI, neither metabolic syndrome nor its components were associated with incident osteoarthritis in the knee. ${ }^{18}$ The hypothesis of this study was that metabolic syndrome is a risk factor for THR or TKR due to osteoarthritis.

The aim of this large prospective study was to assess whether metabolic syndrome or its components were risk factors independent of BMI for subsequent THR or TKR due to primary osteoarthritis.

\section{Methods}

\section{Study population}

Between 1995 and 1997, all inhabitants of Nord-Trøndelag county, aged $\geq 20$ years, were invited to participate in the second wave of the Nord-Trøndelag Health Study 2 (HUNT2). ${ }^{19}$ The HUNT studies include three population-based studies: HUNT1 (1984-1986), HUNT2 (1995-1997) and HUNT3 (2006-2008). HUNT was initially intended to investigate arterial hypertension, diabetes, quality of life and to screen for tuberculosis. However, its scope expanded over time. ${ }^{19}$ This study included baseline data from HUNT2, as the HUNT1 study did not have information on serum triglycerides and HDL.

A total of 65,237 (69.5\%) individuals accepted the invitation to participate in HUNT2. ${ }^{19}$ From this group, we included 63,617 participants with measurements of all metabolic syndrome components at baseline. Of these, 956 were excluded (Figure 1) due to previous joint replacement in the hip or knee $(n=796)$, missing date of operation $(n=158)$ or emigration during baseline period $(n=2)$. Thus, a total of 62,661 persons (32,990 women and 29,671 men) were included in this study. Each participant contributed person-time from participation date in HUNT2 (between August 1995 and June 1997) until total hip or knee replacement due to osteoarthritis, total hip

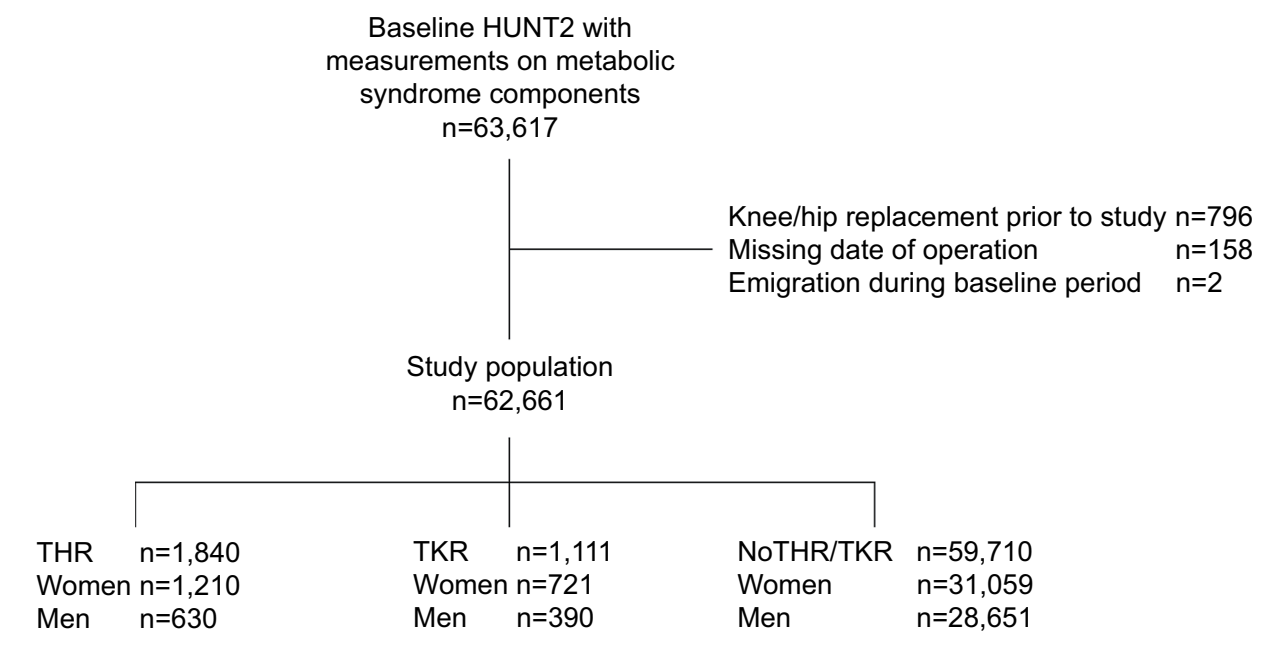

Figure I Flowchart.

Abbreviations: HUNT2, the Nord-Trøndelag Health Study 2; THR, total hip replacement; TKR, total knee replacement. 
or knee replacement due to other causes, migration, death or the end of follow-up (December 31, 2013), whichever occurred first.

\section{Clinical measurements}

The participants were asked to complete a self-administered questionnaire which included a range of health-related questions. Participants were seen once for clinical measurements and blood sampling. The survey included standardized measurement of height, weight, waist circumference and blood pressure by trained nurses or technicians. Weight was measured to the nearest half kilogram with the participants wearing light clothes and no shoes. Waist circumference was measured horizontally at the height of the umbilicus to the nearest centimeter, with the participants standing with their arms hanging relaxed. Blood pressure was measured on the right arm with cuffs adjusted according to the arm circumference, and after the participant had been sitting relaxed for 5 minutes. Measurements based on oscillometry were then taken (Dinamap 845XT; Critikon, Tampa, FL, USA). Systolic and diastolic blood pressure levels were then read three times at 1-minute interval, and the mean of the second and third readings was used in the analysis. Non-fasting blood samples were drawn from each participant. Serum levels of triglycerides, HDL cholesterol and glucose were analyzed on a Hitachi 911 Autoanalyser (Hitachi, Mito, Japan). ${ }^{20}$

According to the Joint Interim Statement, metabolic syndrome is defined as the presence of $\geq 3$ of the following: ${ }^{12}$ waist circumference $\geq 88 \mathrm{~cm}$ in women and $\geq 102 \mathrm{~cm}$ in men, systolic blood pressure $\geq 130 \mathrm{mmHg}$ or diastolic blood pressure $\geq 85 \mathrm{mmHg}$ or use of antihypertensive medication, triglycerides $\geq 1.7 \mathrm{mmol} / \mathrm{L}, \mathrm{HDL}$ cholesterol $<1.3 \mathrm{mmol} / \mathrm{L}$ in women and $<1.0 \mathrm{mmol} / \mathrm{L}$ in men, and glucose $>5.6 \mathrm{mmol} / \mathrm{L}$ or self-reported diabetes. This definition is based on fasting blood samples. When these were lacking, we used a modified definition of metabolic syndrome also used in previous studies, ${ }^{21,22}$ categorizing elevated glucose as serum glucose $\geq 11.1 \mathrm{mmol} / \mathrm{L}$. This is, however, likely to be a stricter cutoff, since it is intended to identify undiagnosed diabetes. ${ }^{23}$ The self-reported diagnosis of diabetes in the HUNT study has been validated in a separate study, demonstrating that $96.4 \%$ of self-reported diabetes could be verified in medical files. ${ }^{24}$

To reduce potential confounding, covariates associated with both metabolic syndrome and joint replacement due to osteoarthritis were adjusted for. These covariates included: age (stratified), gender (female/male), BMI (continuous), current smoking status (never, former, current), physical activity (light, medium, hard) and education (primary, secondary, post-secondary). Cardiovascular disease was evaluated to be a mediator, and was therefore not included as a confounder as this could have biased the analyses. The main analysis was done stepwise; the first model was only adjusted for gender and BMI (Model 1) and the second was fully adjusted (Model 2). Age was stratified into the age groups of $<50,50-69.9$ and $\geq 70$ years at baseline. Physical activity was categorized by duration of light (not sweating or out of breath) physical activity (none, $<1,1-2, \geq 3$ hours/ week) and/or duration of hard (sweating or out of breath) physical activity (none, $<1,1-2, \geq 3$ hours/week). The physical activity questions have previously been validated among men between 20 and 39 years. ${ }^{25}$ This showed acceptable repeatability and validity for the "hard" physical activity questions, but poor validity for the light questions. The two physical activity variables were combined into one variable indicating intensity and duration: none (no activity), medium ( $\leq 2$ hours/week light physical activity and/or $<1$ hour/week hard physical activity) or hard ( $\geq 3$ hours/week light physical activity and/or $\geq 1$ hour/week hard physical activity). Education was defined as the highest level of completed education (primary/vocational, secondary or post-secondary).

To account for potential change in exposure during follow-up, a sensitivity analysis was performed in those who participated in both HUNT2 (1995-1997) and HUNT3 (2006-2008), with $n=30,651$. By excluding those who changed exposure group between HUNT2 and HUNT3, we were able to do an analysis with a lower risk of misclassification of the exposures.

As very few of those between 20 and 30 years at baseline were expected to have a primary THR or TKR due to osteoarthritis, a separate sensitivity analysis also excluded those $<30$ years in the age group $<50$ years. Finally, we also did an analysis stratified on both gender and age to investigate any differences between genders.

\section{Outcome}

This study used THR or TKR due to primary osteoarthritis as the outcome. The unique 11-digit identification number of every Norwegian citizen enabled linkage of HUNT data to the Norwegian Arthroplasty Register (NAR). NAR was established in 1987 and includes all artificial joints from 1994 onward. The completeness of THR and TKR registration is over $95 \%{ }^{26}$ For each arthroplasty performed, the orthopedic surgeon submits a standardized form containing information about the patient, the diagnosis that led to the arthroplasty, the procedure and the type of implant used. ${ }^{27}$ In this paper, primary THR or TKR in patients with primary 
or idiopathic osteoarthritis is considered to be an indicator of severe osteoarthritis.

\section{Statistical methods}

Cox proportional hazards regression models were used to estimate hazard ratios (HRs) with 95\% CIs for metabolic syndrome, and its components, for the first recorded primary THR or TKR due to osteoarthritis. Tests of proportional hazards assumption were evaluated by Schoenfeld residuals and log-minus-log plots (Table S1) and were satisfied for all variables, except for age. The analyses were, therefore, stratified into age groups $(<50,50-69.9$ and $\geq 70$ years $)$ and adjusted for gender, BMI, smoking, physical activity and education. The analyses were performed using Stata 14/IC (StataCorp LP, College Station, TX, USA).

\section{Ethics approval}

This study was approved by the Norwegian Regional Committee for Ethics in Medical Research (REK Sør-Øst C).

\section{Results}

Of the 62,661 participants included in this study, 12,593 (20.1\%) were identified as having metabolic syndrome using the modified definition from Joint Interim Statement with a cutoff for non-fasting blood glucose of $\geq 11.1 \mathrm{mmol} / \mathrm{L}$. The most prevalent components in persons with metabolic syndrome were hypertension, increased triglycerides and low HDL. Members of this group were generally older, had higher BMI, were less physically active, had lower levels of education and higher prevalence of cardiovascular disease than those without metabolic syndrome

Table I Baseline characteristics

\begin{tabular}{|c|c|c|c|c|c|c|}
\hline & \multicolumn{2}{|l|}{ Women } & \multicolumn{2}{|l|}{ Men } & \multicolumn{2}{|l|}{ Total } \\
\hline & MetS, n (\%) & No MetS, n (\%) & MetS, n (\%) & No MetS, n (\%) & MetS, n (\%) & No MetS, n (\%) \\
\hline \multicolumn{7}{|l|}{ Age, years } \\
\hline $19-29$ & $301(4.3)$ & 4,327 (16.7) & $390(7.0)$ & $3,661(15.2)$ & 691 (5.5) & $7,988(16.0)$ \\
\hline $30-39$ & $563(8.0)$ & $5,392(20.8)$ & $727(13.1)$ & 4,623 (19.2) & $1,290(10.2)$ & $10,015(20.0)$ \\
\hline $40-49$ & I,037 (I4.7) & $5,952(22.9)$ & I,084 (19.5) & $5,372(22.3)$ & $2,121(16.8)$ & II,324 (22.6) \\
\hline $50-59$ & I,287 (I8.3) & 4,337 (16.7) & I,058 (19.0) & 4,203 (17.4) & 2,345 (18.7) & $8,540(17.1)$ \\
\hline $60-69$ & I,558 (22.2) & $2,9 \mid 1(11.2)$ & I,038 (I8.7) & $3,148(13.0)$ & $2,596(20.6)$ & $6,059(12.1)$ \\
\hline 70-79 & $1,663(23.6)$ & $2,280(8.8)$ & $956(17.2)$ & $2,434(10.1)$ & $2,619(20.8)$ & $4,7 \mid 4(9.4)$ \\
\hline$\geq 80$ & $626(8.9)$ & 756 (2.9) & $305(5.5)$ & $672(2.8)$ & $931(7.4)$ & I,428 (2.8) \\
\hline \multicolumn{7}{|l|}{ Gender } \\
\hline Women & & & & & 7,035 (55.9) & 25,955 (5I.8) \\
\hline \multicolumn{7}{|l|}{ BMI, kg/m² } \\
\hline$<18.5$ & $7(0.1)$ & $328(1.3)$ & $4(0.1)$ & $109(0.5)$ & II (0.I) & $437(0.9)$ \\
\hline $18.5-24.99$ & $696(9.9)$ & 13,739 (52.9) & $499(9.0)$ & $9,853(40.8)$ & $\mathrm{I}, 195$ (9.5) & $23,592(47.1)$ \\
\hline $25-29.99$ & 2,794 (39.7) & $9,400(36.2)$ & $2,710(48.7)$ & $12,244(50.8)$ & $5,504(43.7)$ & $21,644(43.2)$ \\
\hline$\geq 30$ & $3,538(50.3)$ & $2,488(9.6)$ & $2,345(42.2)$ & I,907 (7.9) & $5,883(46.7)$ & $4,395(8.8)$ \\
\hline \multicolumn{7}{|l|}{ Smoking } \\
\hline Never & $3,536(52.1)$ & I I,958 (47.I) & I,7I5 (3I.5) & $9,274(39.1)$ & $5,25 I(42.9)$ & $21,232(43.2)$ \\
\hline Former & I,492 (22.0) & $5,546(21.8)$ & $2,305(42.4)$ & $7,457(31.4)$ & $3,797(31.1)$ & $13,003(26.5)$ \\
\hline Current & I,763 (25.9) & $7,899(31.1)$ & I,4I9 (26.I) & 7,007 (29.5) & $3,182(26.0)$ & $14,906(30.3)$ \\
\hline Missing & 244 & 552 & 119 & 375 & 363 & 927 \\
\hline \multicolumn{7}{|l|}{ Physical activity } \\
\hline None & 791 (14.4) & $\mathrm{I}, 442(6.1)$ & 575 (II.7) & I,620 (7.3) & $1,366(13.1)$ & $3,062(6.7)$ \\
\hline Medium & $3,169(57.7)$ & $12,749(54.3)$ & $2,623(53.2)$ & $10,375(46.5)$ & $5,792(55.6)$ & $23,124(50.5)$ \\
\hline Hard & I,530 (27.9) & $9,291(39.6)$ & $I, 729(35.1)$ & $10,321(46.2)$ & $3,259(31.3)$ & $19,612(42.8)$ \\
\hline Missing & $\mathrm{I}, 545$ & 2,473 & 631 & $\mathrm{I}, 797$ & 2,176 & 4,270 \\
\hline \multicolumn{7}{|l|}{ Education } \\
\hline Primary/vocational & $5,4 \mid 5(86.5)$ & $16,127(64.8)$ & 4,077 (78.7) & $|6,32|(70.5)$ & $9,492(83)$ & $32,448(67.6)$ \\
\hline Secondary & $297(4.7)$ & $3,034(12.2)$ & $315(6.1)$ & $1,969(8.5)$ & $612(5.3)$ & $5,003(10.4)$ \\
\hline Post-secondary & $552(8.8)$ & $5,719(23)$ & $788(15.2)$ & $4,866(21.0)$ & $\mathrm{I}, 340$ (I I. .7) & $10,585(22.0)$ \\
\hline Missing & 771 & $\mathrm{I}, 075$ & 378 & 957 & $\mathrm{I}, 149$ & 2,032 \\
\hline Increased WC & $5,444(77.4)$ & 3,444 (I 3.3) & 2,883 (5I.8) & $I, 22 \mid(5.1)$ & $8,327(66.1)$ & $4,665(9.3)$ \\
\hline Hypertension & 6,439 (9l.5) & $|I, 77|(45.4)$ & $5,393(97)$ & $16,208(67.2)$ & I I,832 (94.0) & 27,979 (55.9) \\
\hline High triglycerides & $6,335(90.1)$ & $4,189(16.1)$ & $5,383(96.9)$ & $9,083(37.7)$ & II,7I8 (93.I) & $13,272(26.5)$ \\
\hline Low HDL & 5,019 (7I.3) & $4,040(15.6)$ & 3,705 (66.7) & $1,692(7.0)$ & $8,724(69.3)$ & $5,732(11.5)$ \\
\hline IGT or diabetes & 806 (II.5) & $202(0.8)$ & $713(12.8)$ & $333(1.4)$ & $1,519(12.1)$ & $535(I . I)$ \\
\hline
\end{tabular}

Abbreviations: BMI, body mass index; HDL, high-density lipoprotein; IGT, impaired glucose tolerance; MetS, metabolic syndrome; WC, waist circumference. 
(Table 1). At baseline, women and men had a mean age of 49.9 years (SD 17.2) and 49.7 years (SD 16.7), respectively. Correspondingly, mean age at joint replacement was 69.9 years (SD 9.3) and 69.0 years (SD 9.2). In total, 1,840 persons received THR (2.9\%), and 1,111 persons received TKR (1.8\%) during a mean follow-up time of 15.4 (SD 4.3) years.

\section{Metabolic syndrome and THR}

No association was found between metabolic syndrome or its individual components and increased risk of THR (Table 2). In the age group $<50$ years, there was a decreased risk of THR in those with the full metabolic syndrome (HR 0.58 , 95\% CI 0.40-0.83). There was no substantial difference in the analyses in Model 1 and the fully adjusted Model 2. However, persons with impaired glucose tolerance or diabetes in the groups 50-69.9 and $\geq 70$ years had a significantly decreased risk of THR, with HR 0.65 (95\% CI 0.36-0.87) and HR 0.30 (95\% CI 0.13-0.67). Participants $\geq 70$ years with hypertension had a decreased risk of THR (HR 0.63, $95 \%$ CI 0.43-0.92). In the youngest age group, $<50$ years, there was also a decreased risk of THR in those with low HDL (HR 0.72, 95\% CI 0.54-0.94).

\section{Metabolic syndrome and TKR}

Metabolic syndrome was not associated with the risk ofTKR. High waist circumference increased the risk of TKR in the age groups $<50$ years (HR 1.62, 95\% CI 1.10-2.39) and 50-69.9 years (HR 1.43, 95\% CI 1.14-1.80), as shown in Table 2. Hypertension significantly increased the risk of TKR in the age group $<50$ years (HR 1.38, 95\% CI 1.05-1.81).
Apart from these findings, none of the other components of metabolic syndrome were associated with increased risk of TKR. However, low HDL was associated with decreased risk of TKR in both those $<50$ years (HR $0.67,95 \%$ CI $0.49-0.92$ ) and $\geq 70$ years (HR $0.53,95 \%$ CI $0.33-0.86$ ).

\section{Additional analysis}

Of the 30,651 persons who participated in both HUNT2 (1995-1997) and HUNT3 (2006-2008), 31.8\% changed the exposure group during the follow-up period from normal to increased waist circumference and $16.2 \%$ changed the exposure group during the follow-up period from no metabolic syndrome to metabolic syndrome (Table 3 ). When analyzing only those who had not changed the exposure group in each category, there was no longer a decreased risk of THR in participants $<50$ years with metabolic syndrome or low HDL (Figure 2). In this analysis, increased waist circumference was

Table 3 Number of participants who switched the exposure groups during follow-up, including only those who participated in both HUNT2 (1995-1997) and HUNT3 (2006-2008) (N=30,65I)

\begin{tabular}{lll}
\hline & $\begin{array}{l}\text { From unexposed } \\
\text { to exposed group, } \\
\mathbf{n}(\%)\end{array}$ & $\begin{array}{l}\text { From exposed to } \\
\text { unexposed group, } \\
\mathbf{n}(\%)\end{array}$ \\
\hline Waist circumference & $9735(31.8)$ & $274(0.9)$ \\
Hypertension & $3955(12.9)$ & $3303(10.8)$ \\
High triglycerides & $4767(15.6)$ & $4296(14.0)$ \\
Low HDL & $3175(10.4)$ & $2331(7.6)$ \\
IGT or diabetes & $1115(3.6)$ & $13(0.04)$ \\
Metabolic syndrome & $4971(16.2)$ & $1428(4.7)$ \\
\hline
\end{tabular}

Abbreviations: HDL, high-density lipoprotein; HUNT2, the Nord-Trøndelag Health Study 2; IGT, impaired glucose tolerance.

Table 2 Risk of THR or TKR by metabolic syndrome components and metabolic syndrome

\begin{tabular}{|c|c|c|c|c|c|c|}
\hline & \multicolumn{3}{|l|}{ Model Ia } & \multicolumn{3}{|l|}{ Model $2^{b}$} \\
\hline & $<50$ years & 50-69.9 years & $\geq 70$ years & $<50$ years & 50-69.9 years & $\geq 70$ years \\
\hline & HR (95\% CI) & HR (95\% CI) & HR (95\% CI) & HR (95\% CI) & HR (95\% CI) & HR (95\% CI) \\
\hline \multicolumn{7}{|l|}{ THR } \\
\hline Increased waist circumference & I.25 (0.88-I.77) & $0.98(0.83-1.16)$ & I.I9 (0.90-I.57) & $1.17(0.81-1.68)$ & $\mathrm{I} .08(0.89-1.30)$ & $1.22(0.86-1.73)$ \\
\hline Hypertension & $\mathrm{I} .24(0.98-1.55)$ & $1.16(1.00-1.35)$ & $0.63^{*}(0.46-0.87)$ & I.I3 (0.89-I.43) & $1.09(0.93-1.28)$ & $0.63 *(0.43-0.92)$ \\
\hline High triglycerides & $0.92(0.72-I .17)$ & $0.95(0.84-1.07)$ & $0.86(0.70-1.07)$ & $0.86(0.66-I . I I)$ & $0.93(0.8 \mathrm{I}-\mathrm{I} .07)$ & $0.89(0.69-1.16)$ \\
\hline Low HDL & $0.7 \mid *(0.54-0.93)$ & $0.99(0.86-1.14)$ & $0.82(0.64-1.04)$ & $0.72 *(0.54-0.94)$ & $0.95(0.8 I-I .12)$ & $0.85(0.62-1.15)$ \\
\hline IGT or diabetes & $0.73(0.23-2.29)$ & $0.60 *(0.4 I-0.87)$ & $0.33 *(0.18-0.61)$ & $0.78(0.25-2.44)$ & $0.65 *(0.36-0.87)$ & $0.30 *(0.13-0.67)$ \\
\hline Metabolic syndrome & $0.64 *(0.46-0.90)$ & $0.94(0.8 I-1.09)$ & $0.82(0.65-1.04)$ & $0.58 *(0.40-0.83)$ & $0.93(0.79-1.10)$ & $0.83(0.65-1.14)$ \\
\hline \multicolumn{7}{|l|}{ TKR } \\
\hline Increased waist circumference & $1.62 *(1.12-2.36)$ & $1.43^{*}(1.16-1.76)$ & $1.45(0.98-2.15)$ & $1.62 *(1.10-2.39)$ & $1.43 *(1.14-1.80)$ & $1.55(0.95-2.53)$ \\
\hline Hypertension & $1.44 *(1.11-1.88)$ & $1.12(0.91-1.37)$ & $0.76(0.45-1.30)$ & $1.38^{*}(1.05-1.81)$ & $1.17(0.93-1.47)$ & $0.68(0.37-1.25)$ \\
\hline High triglycerides & $0.97(0.74-1.27)$ & $\mathrm{I} .03(0.88-\mathrm{I} .20)$ & I.I 7 (0.86-I.59) & $0.97(0.73-1.28)$ & $\mathrm{I} .05(0.89-\mathrm{I} .25)$ & $\mathrm{I} .27(0.87-\mathrm{I} .85)$ \\
\hline Low HDL & $0.62 *(0.46-0.84)$ & $0.94(0.78-I .12)$ & $0.66 *(0.46-0.93)$ & $0.67 *(0.49-0.92)$ & $1.04(0.86-1.26)$ & $0.53^{*}(0.33-0.86)$ \\
\hline IGT or diabetes & I.0I (0.37-2.73) & $0.73(0.50-1.09)$ & $0.79(0.45-1.40)$ & $0.85(0.27-2.66)$ & $0.70(0.45-I . I I)$ & $0.78(0.38-1.60)$ \\
\hline Metabolic syndrome & $0.94(0.68-1.30)$ & $\mathrm{I} .07$ (0.90-I.28) & $1.25(0.91-1.73)$ & $0.89(0.63-\mathrm{I} .26)$ & $1.16(0.96-1.41)$ & $1.27(0.85-1.90)$ \\
\hline
\end{tabular}

Notes: *Significant at $p<0.05$. aModel I, HR adjusted for gender and BMI. bModel 2, HR adjusted for gender, BMI, smoking, physical activity and education.

Abbreviations: BMI, body mass index; HDL, high-density lipoprotein; HR, hazard ratio; IGT, impaired glucose tolerance; THR, total hip replacement; TKR, total knee replacement. 


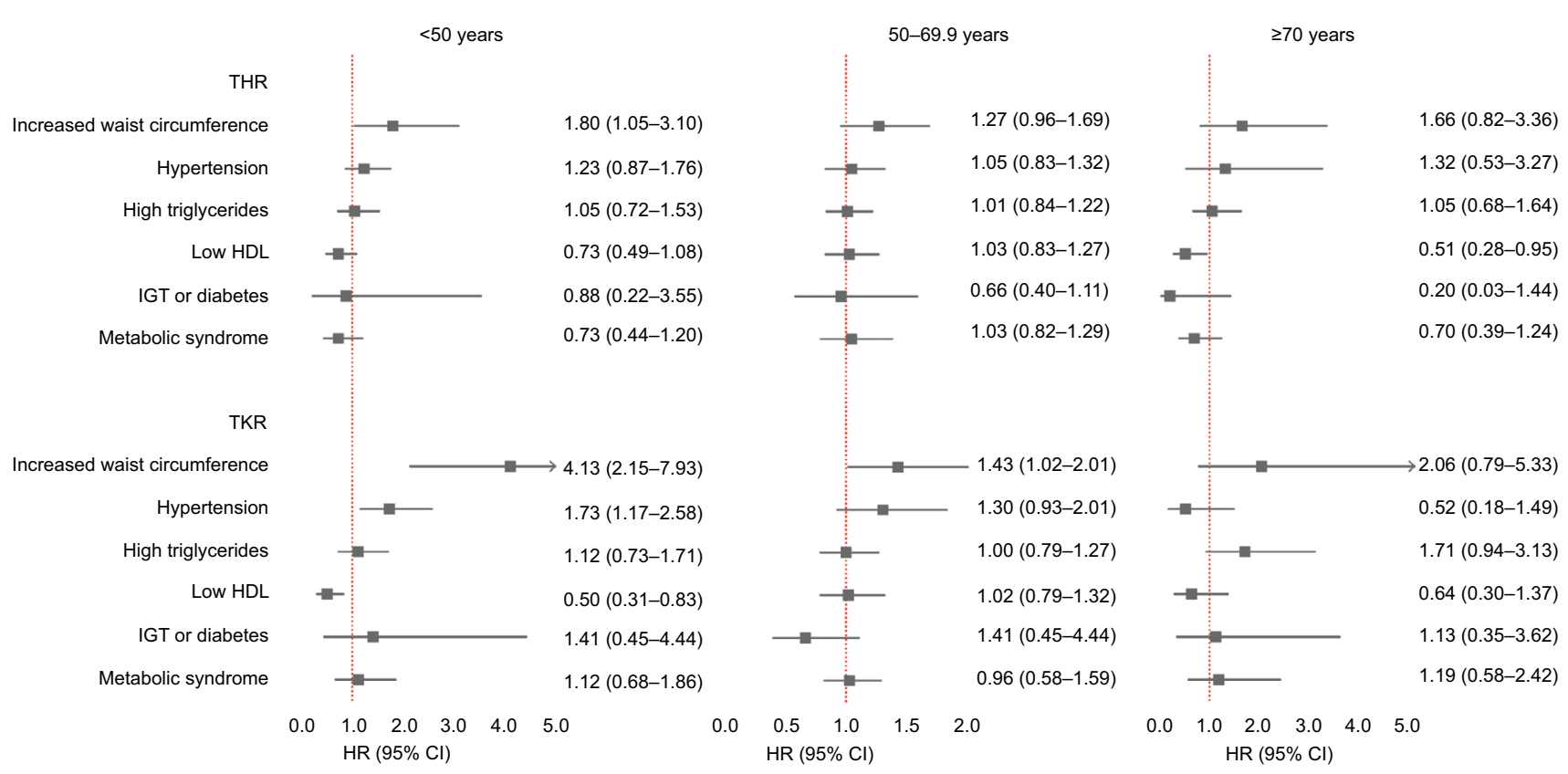

Figure 2 Risk of THR or TKR by metabolic syndrome components and metabolic syndrome including only those patients who did not change exposure groups during follow-up.

Note: HRs adjusted for gender and BMI.

Abbreviations: BMI, body mass index; HDL, high-density lipoprotein; HR, hazard ratio; IGT, impaired glucose tolerance; THR, total hip replacement; TKR, total knee replacement.

also found to be a risk factor for THR in persons $<50$ years (HR 1.80, 95\% CI 1.05-3.10), and there continued to be an increased risk of TKR in the age groups $<50$ years (HR 4.13 , 95\% CI 2.15-7.93) and 50-69.9 years (HR 1.43, 95\% CI 1.02-2.01). In contrast to the main analysis, hypertension was no longer a protective factor for THR in persons $>70$ years, nor was impaired glucose tolerance or diabetes protective for THR in persons between 50 and 69.9 years or $>70$ years of age.

In a separate sensitivity analysis of the age group $<50$ years, participants $<30$ years at baseline were excluded. Hypertension was then only borderline significant (HR 1.29, 95\% CI 0.98-1.70); but apart from this, the results were not substantially different from the main analysis (Figure 3).

When stratifying on both gender and age, we found increased risk of TKR only in men $<50$ years with hypertension (HR 1.90, 95\% CI 1.16-3.11), as shown in Table S2. However, there was still an increased risk of TKR in both men and women between 50 and 69.9 years with increased waist circumference.

\section{Discussion}

In this large prospective study with over 60,000 participants, we found no increased risk of THR or TKR in persons with metabolic syndrome. There was a reduced risk of THR in participants $<50$ years with metabolic syndrome, but this association was no longer significant when exclud- ing those who changed exposure group during follow-up. We found an increased risk of TKR in participants $<70$ years with increased waist circumference and in those $<50$ years with hypertension. Apart from these findings, neither metabolic syndrome nor its components increased the risk of THR or TKR due to osteoarthritis.

In the main analysis (Table 2), we did not find any association between the full metabolic syndrome and THR or TKR, except in persons $<50$ years with metabolic syndrome, who were found to have a decreased risk of THR (HR 0.58, 95\% CI 0.40-0.83). However, our study had information on the development of exposure about 10 years after baseline, and this made it possible to account for changes in the exposure status of metabolic syndrome during follow-up: $16.2 \%$ of those who participated in both HUNT2 and HUNT3 went from unexposed to exposed. This misclassification of exposure could have affected the results. We, therefore, did a sensitivity analysis including only those whose exposure status did not change during the first 10 years of follow-up and found that metabolic syndrome was no longer associated with decreased risk of THR in those $<50$ years. As in the main analysis, in none of the other age strata was metabolic syndrome found to be a risk factor for THR or TKR. We, therefore, conclude that metabolic syndrome is not an important risk factor for THR or TKR independent of BMI. 


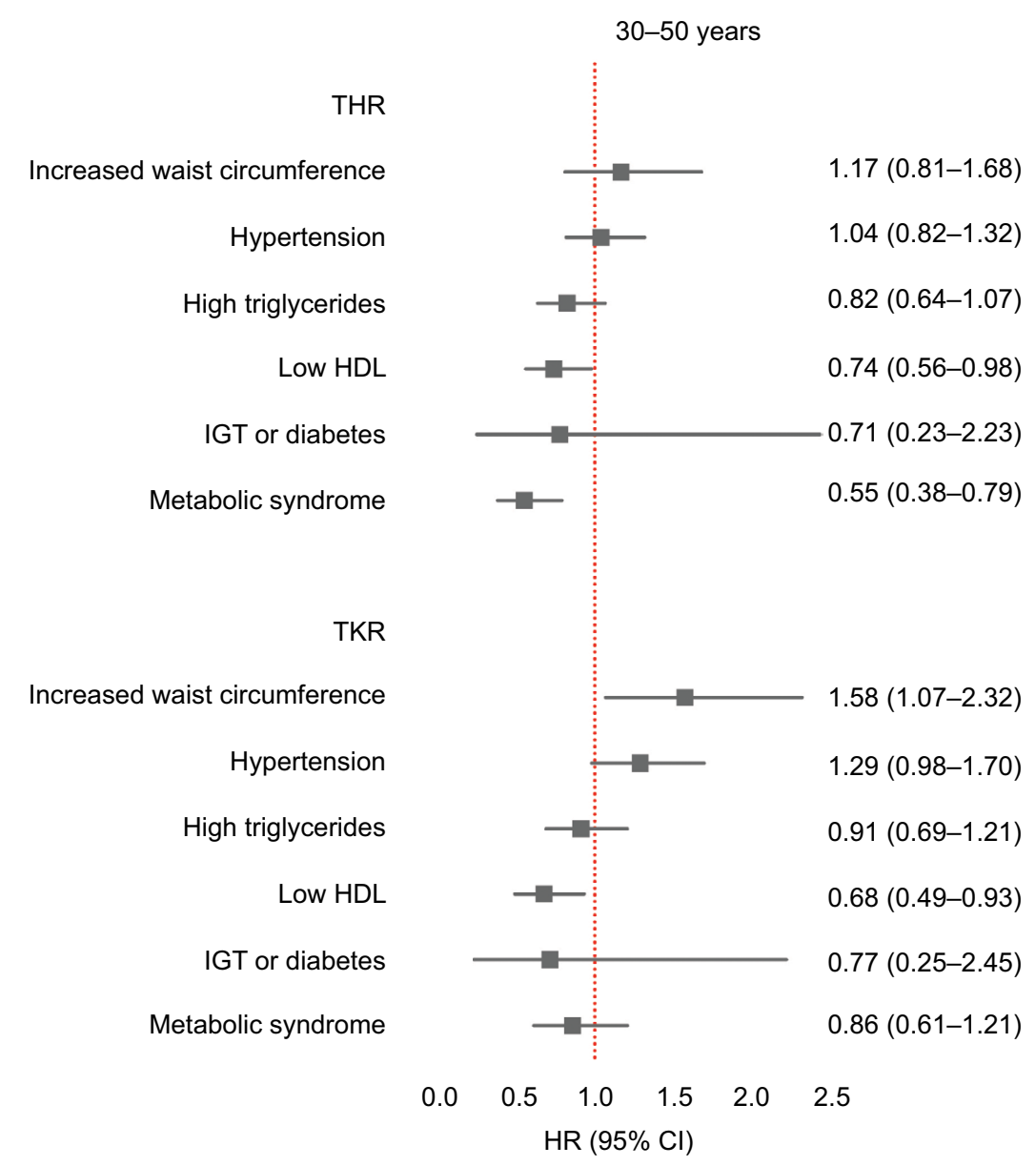

Figure 3 Risk of THR or TKR by metabolic syndrome components and metabolic syndrome in participants $<50$ years after excluding participants $<30$ years at baseline. Note: HRs adjusted for gender, BMI, smoking, physical activity and education.

Abbreviations: BMI, body mass index; HDL, high-density lipoprotein; HR, hazard ratio; IGT, impaired glucose tolerance; THR, total hip replacement; TKR, total knee replacement.

The high number of participants allowed for stratification by age, and thereby the investigation of how some components of metabolic syndrome could have different effects on risk of total joint replacement in younger and older age groups. This could help explain some of the previous conflicting results regarding the association between metabolic syndrome and total joint replacement; what may be a risk factor in those $<50$ years, such as hypertension or increased waist circumference, may not be a risk factor in those $\geq 70$ years (Table 2). The mechanisms behind this are not clear, but it is possible that components of metabolic syndrome could be seen as a relative contraindication to joint replacement surgery to a higher degree in the old, compared to the young. Thus, these components could be protective against surgery, but not necessarily osteoarthritis, in the old. Using THR and TKR as indicators of osteoarthritis had the advantage of being an unambiguous indicator of severe disease burden compared to other osteoarthritis definitions, for example, radiographic criteria, symptom criteria or osteoarthritis defined by self-reported diagnosis. ${ }^{28}$ Using total joint replacement as an endpoint for osteoarthritis also helps distinguish between severe disease and common minor disability. ${ }^{29}$ However, there are several important limitations to this approach. Firstly, persons with moderate osteoarthritis who engage in demanding physical activities could be more motivated to have surgery than less active persons. Secondly, the metabolic syndrome risk factors could influence the orthopedic surgeon's choice regarding treatment, giving a healthy patient selection bias with corresponding underestimation of the effect of possible risk factors. This effect could have been what we observed when we observed that hypertension and increased waist circumference were not found to be risk factors in those $\geq 70$ years. Our findings are also in line with a study by Nielen et al which found that risk of severe osteoarthritis necessitating THR or TKR decreased with increasing severity of diabetes mellitus. ${ }^{30}$ This could help explain the apparent protective effect of impaired glucose tolerance or diabetes in the two older age groups. 
We found an increased risk of TKR in participants $<50$ years with hypertension, and this effect was strongest in men. Hypertension as a risk factor for TKR is consistent with previous findings by Monira Hussain et al. ${ }^{15}$ In both the Chingford study from the UK and the ROAD study from Japan, hypertension was found to be associated with osteoarthritis of the knee, independent of BMI. ${ }^{17,31}$ A recent study by Niu et al reported that diastolic blood pressure was related to incident symptomatic osteoarthritis. ${ }^{18}$ The prevalence of atherosclerotic risk factors, which include hypertension, has been reported to be higher in individuals with osteoarthritis. ${ }^{32}$ It has been hypothesized that vascular pathology of subchondral small vessels could lead to local ischemia and subsequent development of osteoarthritis. ${ }^{33,34}$ Le Clanche et al summarized a possible pathologic pathway between hypertension and osteoarthritis in a recent review ${ }^{35}$ and attributed the connection to a reduced capacity of cells to produce nitric oxide, as hypertension causes a narrowing of the blood vessels. ${ }^{36}$ This again leads to reduced blood flow in the subchondral bone, and thereby a compromised exchange of nutrients and oxygen and degradation of cartilage. ${ }^{34}$ This subchondral ischemia could also induce osteocyte apoptosis in the subchondral bone, which again could lead to osteoclast recruitment and subchondral bone loss. ${ }^{37}$

Persons $<70$ years with increased waist circumference had an increased risk of TKR. Our results were concordant with several previous studies reporting increased central obesity to be a risk factor for osteoarthritis after adjustment for BMI. ${ }^{4,1538}$ It may be that the increased amount of abdominal fat tissue releases inflammatory mediators (adipokines, free fatty acids, reactive oxygen species) that, in turn, affect the joints and cartilage. ${ }^{13,17,39}$ Another explanation could be that a high BMI may be due to either a large muscle/skeletal mass or a large amount of fat tissue. Overweight due to a large muscle mass could, therefore, be less harmful for the knees than overweight due to a large amount of abdominal fat tissue. Waist circumference may, therefore, differentiate these two groups.

\section{Strengths and limitations}

To the best of our knowledge, this is the largest prospective population study addressing the association between metabolic syndrome and total joint replacement due to primary osteoarthritis. In most cases, the metabolic syndrome components were measured many years prior to joint replacement. The ability to adjust for multiple potential confounders was a strength in this study. Even though the participation rate in HUNT2 was fairly high compared to most other surveys, there is always a potential for selection bias. ${ }^{20}$ In particular, men from young age groups had a lower participation rate. However, since primary THR and TKR are most common in the elderly population, the effect of this selection bias in our study population should be minimal.

Information on potential change in exposure group during follow-up was also a strength in this study. When excluding participants who switched the exposure group during the first 10 years of follow-up, there was no longer an association between hypertension and THR in persons $>70$ years, nor was impaired glucose tolerance or diabetes associated with THR in persons between 50 and 69.9 years or $>70$ years. The apparent protective effects of these exposures in the main analyses could, therefore, have been due to misclassification of exposure.

When analyzing women and men separately, we found that increased waist circumference was a risk factor for TKR in both genders. However, only men with hypertension had an increased risk of TKR. The reason for this difference is not clear, as one would expect that an underlying biologic mechanism of subchondral ischemia was the same in both genders. Further studies on a possible gender difference in hypertension and knee osteoarthritis are, therefore, warranted.

THR or TKR is very uncommon in persons $<30$ years, and including this group in the analysis could have distorted the results. In a sensitivity analysis excluding those $<30$ years, we found that the risk of TKR in participants with hypertension was weakened (HR 1.29, 95\% CI 0.98-1.70). This could indicate that including young participants could lead to overestimation of the effect of hypertension on TKR, and this should be taken into account in further studies.

Participants taking antihypertensive medication and/ or with known diabetes were accounted for in the analysis by including them in the hypertensive and impaired glucose tolerance groups, respectively. However, we did not have information on cholesterol-lowering medication, and this could have resulted in differential misclassification of exposure as persons on medication were classified as having normal serum levels, thus potentially weakening the association between HDL/triglycerides and joint replacement. This possible misclassification may explain the reduced risk of THR or TKR in persons with low HDL in both the highest and lowest age strata (Table 2; Figure 2).

As previously described, a limitation in this study was that we only had information on non-fasting serum blood glucose. Our cutoff level of serum glucose $\geq 11.1 \mathrm{mmol} / \mathrm{L}$ is likely to be a stricter definition of impaired glucose tolerance, ${ }^{23}$ and may thus have resulted in an underestimation of 
any association between impaired glucose tolerance/diabetes and joint replacement.

Many participants may have had metabolic syndrome for some time before entering the study. This could have led to a bias in estimation resulting from studying prevalent exposure rather than new exposure. ${ }^{40} \mathrm{We}$ were not able to differentiate between new and prevalent cases, and thus, the effect of this prevalent cohort bias could have led to an underestimation of any association between metabolic syndrome and total joint replacement.

Waist circumference and BMI are correlated. Estimating the correlation between waist circumference (dichotomous) and BMI (continuous) gave an $R^{2}$ of 0.38 in the age group $<50$ years, 0.43 in the age group 50-69.9 years and 0.42 in the age group $\geq 70$ years. This corresponded to a variance inflation factor (VIF) of 1.62, 1.75 and 1.72, respectively. VIF estimates how much the variance of waist circumference is inflated because of dependence on BMI. Thus, a VIF of 1.62 indicates that the variance of waist circumference is $62 \%$ larger than it would be if it was completely unrelated to BMI. There are different opinions on when the correlation is high enough to become a problem, but a VIF $<4$ may be acceptable. ${ }^{41}$ However, it is difficult to exactly estimate the effect of increased waist circumference, independent of BMI.

Education is used as an indicator of socioeconomic status, and the HRs did not change significantly after adjusting for this factor. In addition to this, the hospital care in Norway is publicly financed and free of charge for patients. Therefore, we do not think that socioeconomic factors represented a major confounder in this material.

Previous injuries increase the risk of osteoarthritis, especially in the knee. ${ }^{42,43}$ Even though we did not have direct information on previous injury, the operating surgeon had to report whether the knee joint replacement was due to primary/ idiopathic osteoarthritis or a sequela from fracture, ligament injury, meniscal injury, infection, rheumatoid arthritis or ankylosing spondylitis. We only included joint replacement due to primary/idiopathic osteoarthritis.

Validation of diagnoses from the NAR has only been done for young adults $<40$ years of age undergoing THR due to hip dysplasia, ${ }^{44}$ and is therefore inapplicable to our study population. However, numbers from the Danish Hip Arthroplasty Registry show a positive predictive value of $85 \%$ regarding the primary hip osteoarthritis diagnosis. ${ }^{45}$ The results from the Danish registry are probably comparable to the Norwegian registry.

The main clinical implication of this study was that we did not find any increased risk of osteoarthritis in participants with metabolic syndrome. Metabolic syndrome may, therefore, not be an effective screening tool for identifying individuals with increased risk of THR or TKR. It is, however, possible to identify two groups that should receive special attention in reducing the risk of TKR due to osteoarthritis: persons $<70$ years with increased waist circumference and persons $<50$ years with hypertension. The clinical focus should still be mainly on weight reduction, as this is also an important first step in the treatment and prevention of hypertension. ${ }^{46}$ However, treatment for the other metabolic syndrome components is, of course, still advisable due to their association with increased risk of cardiovascular disease.

\section{Conclusion}

This study found an increased risk of TKR in men $<50$ years with hypertension and in persons $<70$ years with increased waist circumference. Apart from this, neither metabolic syndrome nor its components were associated with increased risk of THR or TKR due to primary osteoarthritis.

\section{Acknowledgments}

The Nord-Trøndelag Health Study is a collaborative effort of the Faculty of Medicine, Norwegian University of Science and Technology; the Norwegian Institute of Public Health and the Nord-Trøndelag County Council. The Norwegian Arthroplasty Register is owned by the Norwegian Orthopedic Association and administered by the Orthopedic Department at Haukeland University Hospital, Bergen, Norway. This study was supported by research grants from the Liaison Committee between the Central Norway Regional Health Authority (RHA) and the Norwegian University of Science and Technology (NTNU), the Dr Egil Kjeldaas foundation (Lege Egil Kjeldaas legat), the Norwegian Orthopaedic Association and from Levanger Hospital, Nord-Trøndelag Hospital Trust. The funding sources had no involvement in the study design, collection, analysis or interpretation of data, writing the paper or the decision to submit the paper for publication.

\section{Author contributions}

AIH participated in the study concept and design, obtained funding, performed the analysis, interpreted the data and drafted the manuscript. LN, MBJ, AL, GF, OF, KS and JAZ were involved in the conception and design of the study. OF was also involved in data collection of THR and TKR. VB contributed with statistical expertise. All authors revised the manuscript for important intellectual content and approved the final version of the manuscript. 


\section{Disclosure}

The authors report no conflicts of interest in this work.

\section{References}

1. Vos T, Flaxman AD, Naghavi M, et al. Years lived with disability (YLDs) for 1160 sequelae of 289 diseases and injuries 1990-2010: a systematic analysis for the Global Burden of Disease Study 2010. Lancet. 2012;380(9859):2163-2196.

2. Apold H, Meyer HE, Nordsletten L, Furnes O, Baste V, Flugsrud GB. Risk factors for knee replacement due to primary osteoarthritis, a population based, prospective cohort study of 315,495 individuals. BMC Musculoskelet Disord. 2014;15(1):217.

3. Felson DT, Anderson JJ, Naimark A, Walker AM, Meenan RF. Obesity and knee osteoarthritis. The Framingham Study. Ann Intern Med. 1988;109(1):18-24.

4. Lohmander LS, Gerhardsson de Verdier M, Rollof J, Nilsson PM, Engstrom G. Incidence of severe knee and hip osteoarthritis in relation to different measures of body mass: a population-based prospective cohort study. Ann Rheum Dis. 2009;68(4):490-496.

5. Apold H, Meyer HE, Espehaug B, Nordsletten L, Havelin LI, Flugsrud GB. Weight gain and the risk of total hip replacement a population-based prospective cohort study of 265,725 individuals. OsteoarthritisCartilage. 2011;19(7):809-815.

6. Flugsrud GB, Nordsletten L, Espehaug B, Havelin LI, Engeland A, Meyer HE. The impact of body mass index on later total hip arthroplasty for primary osteoarthritis: a cohort study in 1.2 million persons. Arthritis Rheum. 2006;54(3):802-807.

7. Flugsrud GB, Nordsletten L, Espehaug B, Havelin LI, Meyer HE. Risk factors for total hip replacement due to primary osteoarthritis: a cohort study in 50,034 persons. Arthritis Rheum. 2002;46(3):675-682.

8. Rai MF, Sandell LJ. Inflammatory mediators: tracing links between obesity and osteoarthritis. Crit Rev Eukaryot Gene Expr. 2011;21(2):131-142.

9. Lago F, Gomez R, Conde J, Scotece M, Gomez-Reino JJ, Gualillo O. Cardiometabolic comorbidities and rheumatic diseases: focus on the role of fat mass and adipokines. Arthritis Care Res (Hoboken). 2011;63(8):1083-1090.

10. Sellam J, Berenbaum F. Is osteoarthritis a metabolic disease? Joint Bone Spine. 2013;80(6):568-573.

11. Zhuo Q, Yang W, Chen J, Wang Y. Metabolic syndrome meets osteoarthritis. Nat Rev Rheumatol. 2012;8(12):729-737.

12. Alberti KG, Eckel RH, Grundy SM, et al; International Association for the Study of Obesity. Harmonizing the metabolic syndrome: a joint interim statement of the International Diabetes Federation Task Force on Epidemiology and Prevention; National Heart, Lung, and Blood Institute; American Heart Association; World Heart Federation; International Atherosclerosis Society; and International Association for the Study of Obesity. Circulation. 2009;120(16):1640-1645.

13. Berenbaum F. Osteoarthritis as an inflammatory disease (osteoarthritis is not osteoarthrosis!). Osteoarthritis Cartilage. 2013;21(1):16-21.

14. Hawker GA, Stanaitis I. Osteoarthritis year in review 2014: clinical. Osteoarthritis Cartilage. 2014;22(12):1953-1957.

15. Monira Hussain S, Wang Y, Cicuttini FM, et al. Incidence of total knee and hip replacement for osteoarthritis in relation to the metabolic syndrome and its components: a prospective cohort study. Semin Arthritis Rheum. 2014;43(4):429-436.

16. Engstrom G, Gerhardsson de Verdier M, Rollof J, Nilsson PM, Lohmander LS. C-reactive protein, metabolic syndrome and incidence of severe hip and knee osteoarthritis. A population-based cohort study. Osteoarthritis Cartilage. 2009;17(2):168-173.

17. Yoshimura N, Muraki S, Oka H, Kawaguchi H, Nakamura K, Akune T. Association of knee osteoarthritis with the accumulation of metabolic risk factors such as overweight, hypertension, dyslipidemia, and impaired glucose tolerance in Japanese men and women: the ROAD study. J Rheumatol. 2011;38(5):921-930.
18. Niu J, Clancy M, Aliabadi P, Vasan R, Felson DT. Metabolic syndrome, its components, and knee osteoarthritis: the framingham osteoarthritis study. Arthritis Rheumatol. 2017;69(6):1194-1203.

19. Krokstad S, Langhammer A, Hveem K, et al. Cohort Profile: the HUNT Study, Norway. Int J Epidemiol. 2013;42(4):968-977.

20. Holmen J, Midthjell K, Krüger Ø, et al. The Nord-Trøndelag Health Study 1995-1997 (HUNT 2): objectives, contents, methods and participation. Norsk Epidemiologi. 2003;13(1):19-32.

21. Moe B, Mork PJ, Holtermann A, Nilsen TI. Occupational physical activity, metabolic syndrome and risk of death from all causes and cardiovascular disease in the HUNT 2 cohort study. Occup Environ Med. 2013;70(2):86-90.

22. Martin RM, Vatten L, Gunnell D, Romundstad P, Nilsen TI. Components of the metabolic syndrome and risk of prostate cancer: the HUNT 2 cohort, Norway. Cancer Causes Control. 2009;20(7):1181-1192.

23. American Diabetes Association. Diagnosis and classification of diabetes mellitus. Diabetes Care. 2012;35(Suppl 1):S64-S71.

24. Midthjell K, Holmen J, Bjorndal A, Lund-Larsen G. Is questionnaire information valid in the study of a chronic disease such as diabetes? The Nord-Trondelag diabetes study. J Epidemiol Community Health. 1992;46(5):537-542.

25. Kurtze N, Rangul V, Hustvedt BE, Flanders WD. Reliability and validity of self-reported physical activity in the Nord-Trøndelag Health Study (HUNT 2). Eur J Epidemiol. 2007;22(6):379-387.

26. Espehaug B, Furnes O, Havelin LI, Engesaeter LB, Vollset SE, Kindseth O. Registration completeness in the Norwegian Arthroplasty Register. Acta Orthop. 2006;77(1):49-56.

27. Havelin LI, Engesaeter LB, Espehaug B, Furnes O, Lie SA, Vollset SE. The Norwegian Arthroplasty Register: 11 years and 73,000 arthroplasties. Acta Orthop Scand. 2000;71(4):337-353.

28. Kim C, Nevitt MC, Niu J, et al. Association of hip pain with radiographic evidence of hip osteoarthritis: diagnostic test study. $B M J$. 2015;351:h5983.

29. Dieppe P. Osteoarthritis: time to shift the paradigm. This includes distinguishing between severe disease and common minor disability. BMJ. 1999;318(7194):1299-1300.

30. Nielen JT, Emans PJ, Dagnelie PC, et al. Severity of diabetes mellitus and total hip or knee replacement: A Population-Based Case-Control Study. Medicine (Baltimore). 2016;95(20):e3739.

31. Yoshimura N, Muraki S, Oka H, et al. Accumulation of metabolic risk factors such as overweight, hypertension, dyslipidaemia, and impaired glucose tolerance raises the risk of occurrence and progression of knee osteoarthritis: a 3-year follow-up of the ROAD study. Osteoarthritis Cartilage. 2012;20(11):1217-1226.

32. Singh G, Miller JD, Lee FH, Pettitt D, Russell MW. Prevalence of cardiovascular disease risk factors among US adults with self-reported osteoarthritis: data from the Third National Health and Nutrition Examination Survey. Am J Manag Care. 2002;8(15 Suppl):S383-S391.

33. Conaghan PG, Vanharanta H, Dieppe PA. Is progressive osteoarthritis an atheromatous vascular disease? Ann Rheum Dis. 2005;64(11): 1539-1541.

34. Findlay DM. Vascular pathology and osteoarthritis. Rheumatology (Oxford). 2007;46(12):1763-1768.

35. Le Clanche S, Bonnefont-Rousselot D, Sari-Ali E, Rannou F, Borderie D. Inter-relations between osteoarthritis and metabolic syndrome: a common link? Biochimie. 2016;121:238-252.

36. Huang PL, Huang Z, Mashimo H, et al. Hypertension in mice lacking the gene for endothelial nitric oxide synthase. Nature. 1995;377(6546):239-242.

37. Aguirre JI, Plotkin LI, Stewart SA, et al. Osteocyte apoptosis is induced by weightlessness in mice and precedes osteoclast recruitment and bone loss. J Bone Miner Res. 2006;21(4):605-615.

38. Wang Y, Simpson JA, Wluka AE, et al. Relationship between body adiposity measures and risk of primary knee and hip replacement for osteoarthritis: a prospective cohort study. Arthritis Res Ther. 2009;11(2):R31. 
39. Courties A, Gualillo O, Berenbaum F, Sellam J. Metabolic stressinduced joint inflammation and osteoarthritis. OsteoarthritisCartilage. 2015;23(11):1955-1965.

40. Heaton B, Applebaum KM, Rothman KJ, et al. The influence of prevalent cohort bias in the association between periodontal disease progression and incident coronary heart disease. Ann Epidemiol. 2014;24(10):741-746.

41. O'Brien RM. A caution regarding rules of thumb for variance inflation factors. Qual Quant. 2007;41(5):673-690.

42. Oiestad BE, Holm I, Engebretsen L, Aune AK, Gunderson R, Risberg MA. The prevalence of patellofemoral osteoarthritis 12 years after anterior cruciate ligament reconstruction. Knee Surg Sports Traumatol Arthrosc. 2013;21(4):942-949.

43. Murphy L, Schwartz TA, Helmick CG, et al. Lifetime risk of symptomatic knee osteoarthritis. Arthritis Rheum. 2008;59(9):1207-1213.
44. Engesaeter IO, Lehmann T, Laborie LB, Lie SA, Rosendahl K, Engesaeter LB. Total hip replacement in young adults with hip dysplasia: age at diagnosis, previous treatment, quality of life, and validation of diagnoses reported to the Norwegian Arthroplasty Register between 1987 and 2007. Acta Orthop. 2011;82(2):149-154.

45. Pedersen A, Johnsen S, Overgaard S, Soballe K, Sorensen HT, Lucht $\mathrm{U}$. Registration in the danish hip arthroplasty registry: completeness of total hip arthroplasties and positive predictive value of registered diagnosis and postoperative complications. Acta Orthop Scand. 2004;75(4):434-441.

46. Neter JE, Stam BE, Kok FJ, Grobbee DE, Geleijnse JM. Influence of weight reduction on blood pressure: a meta-analysis of randomized controlled trials. Hypertension. 2003;42(5):878-884. 


\section{Supplementary materials}

Table SI Graphical evaluation of proportional hazards assumption for Cox regression, using log-minus-log plot for categorical variables and Schoenfeld residual plot for continuous variables
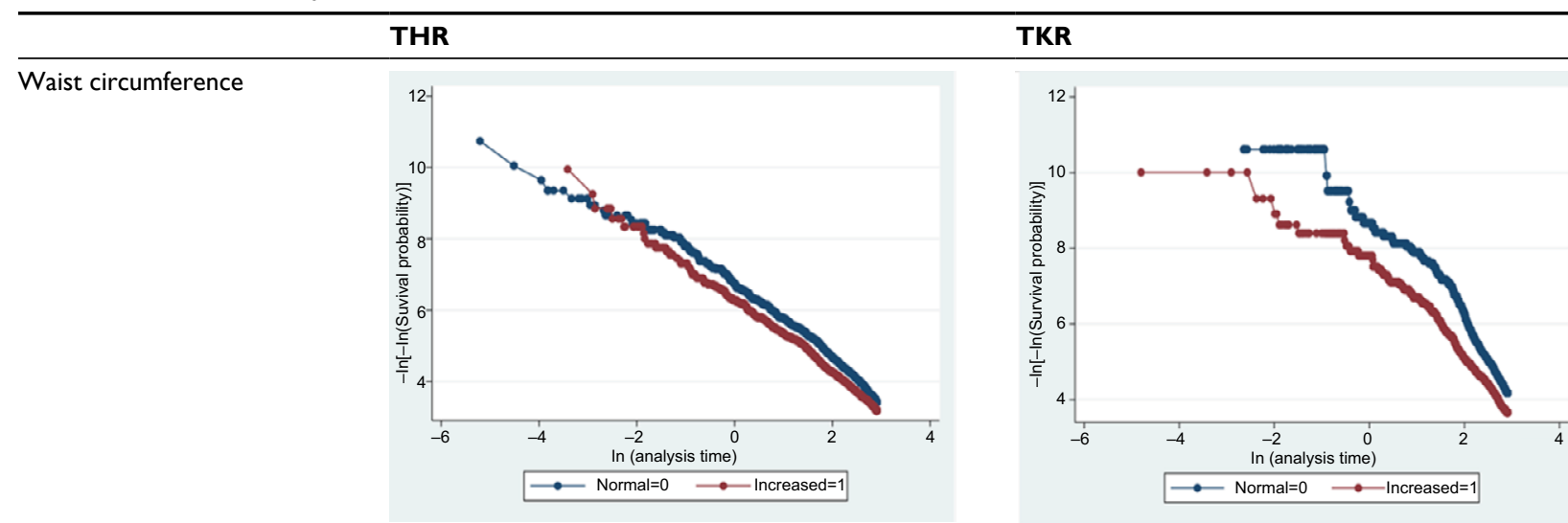

Blood pressure
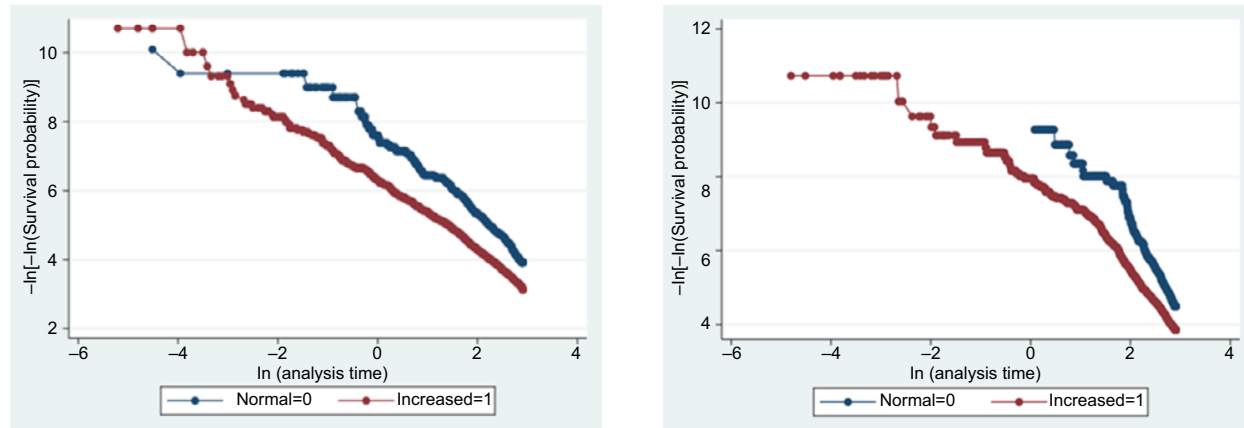

Triglycerides
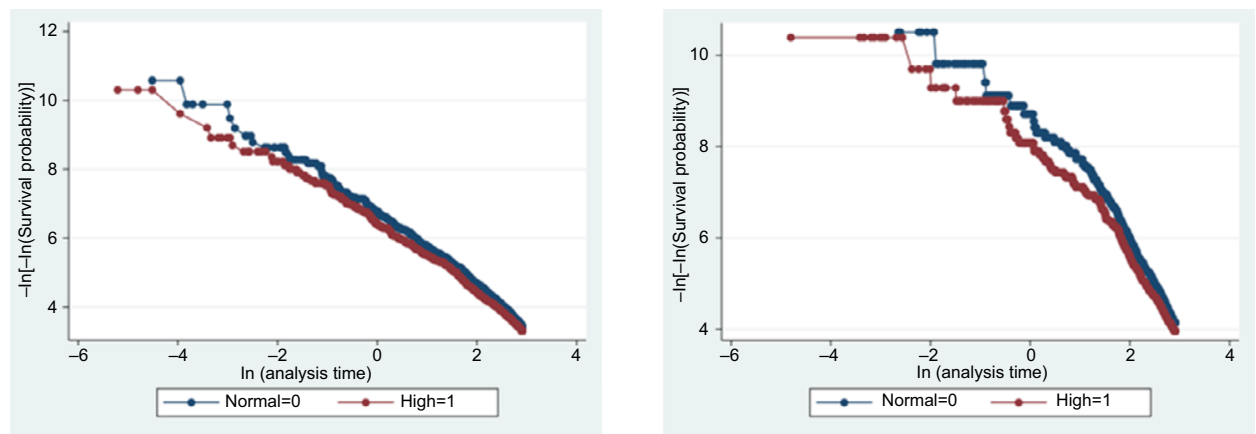

HDL
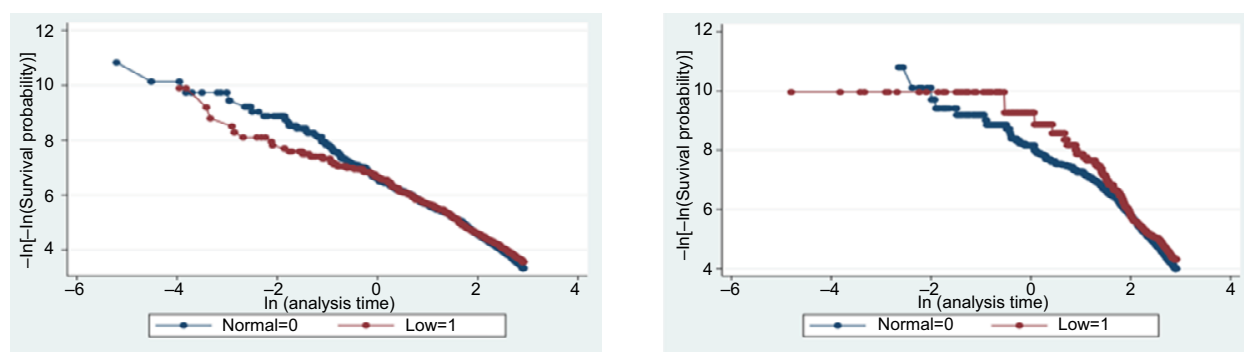

(Continued) 
Table SI (Continued)

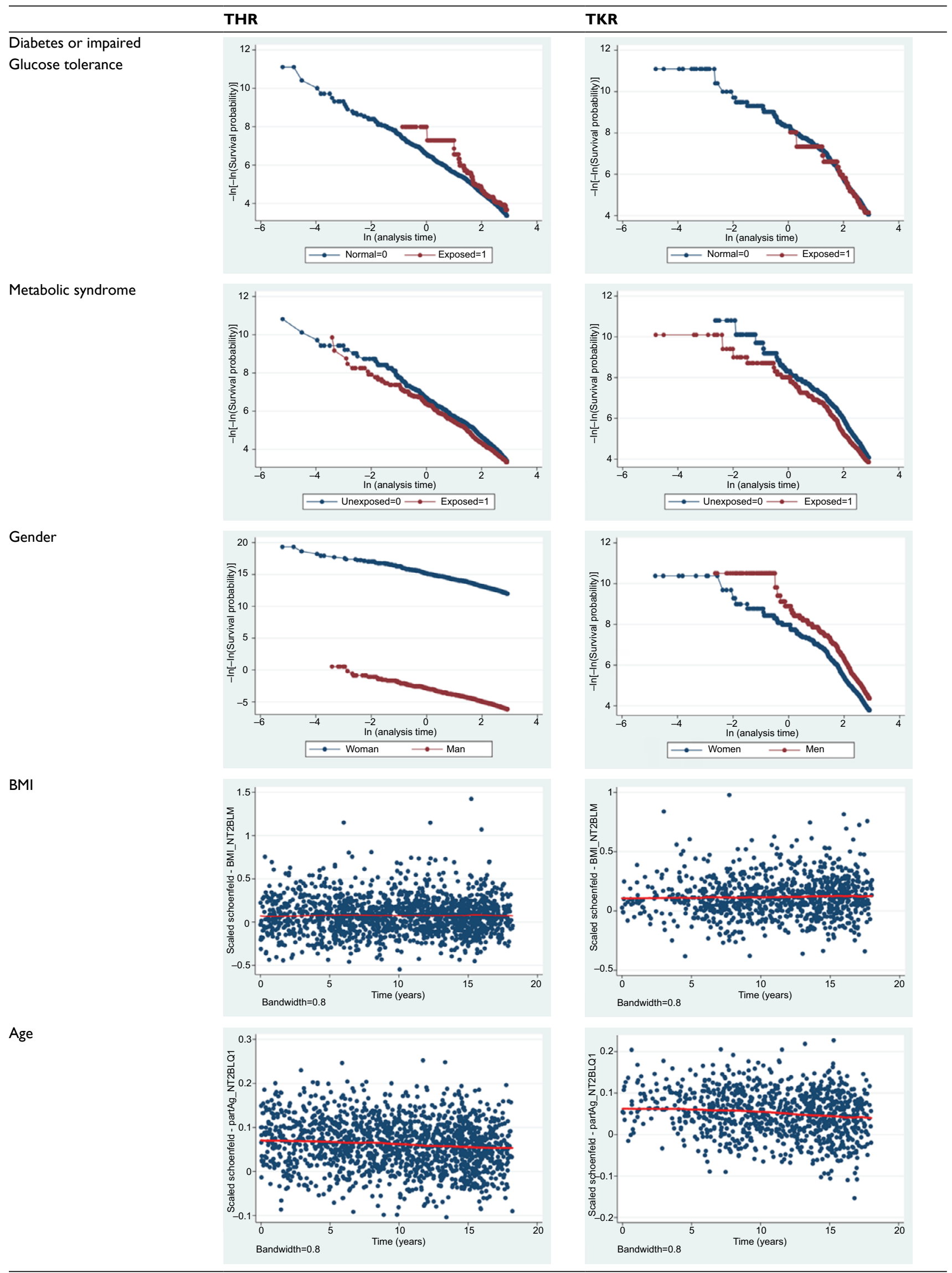

Abbreviation: BMI, body mass index; HDL, high-density lipoprotein; THR, total hip replacement; TKR, total knee replacement. 
Table S2 Risk of THR or TKR by metabolic syndrome components and metabolic syndrome stratified on gender and age ${ }^{\mathrm{a}}$

\begin{tabular}{|c|c|c|c|c|c|c|}
\hline & \multicolumn{3}{|l|}{ Women } & \multicolumn{3}{|l|}{ Men } \\
\hline & $<50$ years & 50-69.9 years & $\geq 70$ years & $<50$ years & 50-69.9 years & $\geq 70$ years \\
\hline & HR (95\% Cl) & HR (95\% Cl) & HR (95\% Cl) & HR (95\% Cl) & HR (95\% Cl) & HR (95\% Cl) \\
\hline \multicolumn{7}{|l|}{ THR } \\
\hline Increased waist circumference & $1.35(0.88-2.08)$ & $1.05(0.83-1.32)$ & $1.25(0.8 \mathrm{I}-\mathrm{I} .92)$ & $0.78(0.39-1.58)$ & I. $15(0.83-1.59)$ & I. $15(0.63-2.09)$ \\
\hline Hypertension & $1.25(0.94-1.66)$ & $1.10(0.90-1.35)$ & $0.54 *(0.33-0.88)$ & $0.92(0.62-1.38)$ & $1.09(0.82-1.45)$ & $0.77(0.42-1.43)$ \\
\hline High triglycerides & $0.94(0.68-1.32)$ & $1.00(0.84-1.19)$ & $1.04(0.74-1.44)$ & $0.74(0.50-1.10)$ & $0.82(0.66-1.01)$ & $0.66(0.43-1.01)$ \\
\hline Low HDL & $0.75(0.54-1.03$ & $0.96(0.79-1.16$ & $0.91(0.63-\mid .31)$ & $0.62(0.36-1.07)$ & $0.93(0.70-1.23)$ & $0.71(0.40-1.25)$ \\
\hline IGT or diabetes & No exposed cases & $0.48 *(0.25-0.93)$ & $0.34 *(0.12-0.92)$ & $2.31(0.73-7.32)$ & $0.65(0.35-1.18)$ & $0.24 *(0.06-0.99)$ \\
\hline Metabolic syndrome & $0.6 \mathrm{I} *(0.39-0.95)$ & $0.96(0.78-1.18)$ & $0.92(0.64-1.32)$ & $0.5 I *(0.27-0.94)$ & $0.86(0.65-1.14)$ & $0.68(0.40-1.16)$ \\
\hline \multicolumn{7}{|l|}{ TKR } \\
\hline Increased waist circumference & I.48 (0.90-2.43) & $1.34 *(1.01-1.78)$ & $1.59(0.86-2.94)$ & $1.76(0.94-3.30)$ & $1.65 *(1.12-2.44)$ & I.5I (0.65-3.50) \\
\hline Hypertension & $1.15(0.81-1.62)$ & $1.09(0.83-1.43)$ & $0.62(0.28-1.37)$ & $1.90 *(1.16-3.11)$ & $1.40(0.92-2.14)$ & $0.80(0.31-2.08)$ \\
\hline High triglycerides & $0.77(0.51-1.16)$ & $1.17(0.94-1.45)$ & $1.26(0.78-2.02)$ & $1.19(0.79-1.80)$ & $0.87(0.65-1.15)$ & $1.35(0.7 \mathrm{I}-2.58)$ \\
\hline Low HDL & $0.69(0.47-1.02)$ & $1.17(0.93-1.48)$ & $0.72(0.43-1.20)$ & $0.62(0.36-1.06)$ & $0.77(0.53-1.12)$ & $0.16 *(0.04-0.68)$ \\
\hline IGT or diabetes & $0.94(0.40-2.18)$ & $0.28^{*}(0.12-0.68)$ & $0.94(0.40-2.18)$ & $0.67(0.09-4.84)$ & I. $38(0.8 \mathrm{I}-2.35)$ & $0.52(0.13-2.17)$ \\
\hline Metabolic syndrome & $0.86(0.54-1.37)$ & $1.28 *(1.01-1.63)$ & $1.46(0.89-2.40)$ & $0.87(0.5 \mathrm{I}-1.47)$ & $0.95(0.67-1.34)$ & I.0I (0.50-2.07) \\
\hline
\end{tabular}

Notes: *Significant at $p<0.05$. a $\mathrm{HRs}$ adjusted for BMI, smoking, education and physical activity.

Abbreviations: BMI, body mass index; HDL, high-density lipoprotein; HR, hazard ratio; IGT, impaired glucose tolerance; THR, total hip replacement; TKR, total knee replacement.

\section{Publish your work in this journal}

Clinical Epidemiology is an international, peer-reviewed, open access, online journal focusing on disease and drug epidemiology, identification of risk factors and screening procedures to develop optimal preventative initiatives and programs. Specific topics include: diagnosis, prognosis, treatment, screening, prevention, risk factor modification,

Submit your manuscript here: https://www.dovepress.com/clinical-epidemiology-journa

\section{Dovepress}

systematic reviews, risk and safety of medical interventions, epidemiology and biostatistical methods, and evaluation of guidelines, translational medicine, health policies and economic evaluations. The manuscript management system is completely online and includes a very quick and fair peer-review system, which is all easy to use. 